RELATO DE CASO

\title{
$\beta$-Talassemia major: um relato de caso
}

\section{$\beta$-Thalassemia major: a case report}

\author{
Ítalo Aguiar Freire ${ }^{1,2}$. Alaíde Maria Rodrigues Deolindo². Mitza Serena Furtado Sanches². Francisco Plácido
} Nogueira Arcanjo ${ }^{2}$.

1 Santa Casa de Misericórdia, Sobral, Ceará, Brasil. 2 Universidade Federal do Ceará (UFC), Sobral, Ceará, Brasil.

\section{RESUMO}

As talassemias configuram doenças relacionadas à síntese deficiente ou ausente de cadeias da hemoglobina, sendo mais comuns as dos tipos $\alpha$ e $\beta$. A forma major é considerada rara e possui uma apresentação clínica ampla, caracterizada por manifestações sistêmicas da anemia grave, como alterações ósseas, hepatoesplenomegalia, cardiopatias, hepatopatias, hipogonadismo, hipotireoidismo, diabetes mellitus, dentre outras. As complicações relacionadas a tais achados se tornam mais evidentes com o retardo do diagnóstico e tratamento inadequado. A raridade de ocorrência na região norte do Ceará e as questões socioeconômicas que envolvem a detecção da doença e condução clínica da paciente pediátrica referida torna importante a devida divulgação na comunidade científica do relato e das questões relacionadas ao tratamento, no intuito de estimular o aprimoramento de políticas públicas que beneficie os pacientes talassêmicos.

Palavras-chave: Talassemia. Anemia hemolítica. Esplenomegalia.

\begin{abstract}
Thalassemias constitute diseases related to the deficient or absent synthesis of hemoglobin chains, being more common the types $\alpha$ and $\beta$. The major form is considered rare and has a broad clinical presentation, characterized by systemic manifestations of severe anemia, such as bone alterations, hepatosplenomegaly, heart diseases, liver diseases, hypogonadism, hypothyroidism, diabetes mellitus, among others. The complications of such findings become more evident with the delay of diagnosis and inadequate treatment. The rarity of occurrence in the northern region of Ceará and the socioeconomic issues that involve the detection of the disease and the clinical conduction of the referred pediatric patient makes important the proper disclosure in the scientific community of the report and treatment-related issues, in order to stimulate the improvement of public policies that benefit thalassemic patients.
\end{abstract}

Keywords: Thalassemia. Hemolytic anemia. Splenomegaly.

Autor correspondente: Ítalo Aguiar Freire, Avenida Comandante Maurocelio, Rua Tarciano da Rocha Pontes, 100, Derby Clube, Sobral, Ceará. CEP: 62042-280. Telefone: +55 88 99951-8570. E-mail: italo.nv@gmail.com

Conflito de interesses: Não há qualquer conflito de interesses por parte de qualquer um dos autores.

Recebido em: 13 Mai 2018; Revisado em: 06 Out 2018; Aceito em: 06 Out 2018. 


\section{INTRODUÇÃO}

As talassemias fazem parte do conjunto de doenças hematológicas hereditárias que resultam em anemia de graus variáveis ocasionadas pelo ritmo anômalo de síntese das cadeias polipeptídicas que compõem a hemoglobina. Os tipos alfa e beta são os mais importantes do ponto de vista de saúde pública e recebem essa classificação a depender da cadeia globínica afetada. ${ }^{1}$

Alterações nos locais de eritropoiese que determinam as talassemias alfa e beta estão associadas às mudanças multigênicas ligadas aos cromossomos 16 e 11, respectivamente. ${ }^{1}$ São descritos cerca de 200 tipos de mutações relacionadas às talassemias da forma beta, sendo cerca de 20 destas responsáveis por $80 \%$ dos casos mundialmente. As mais estudadas são: $\beta 0$ CD 39, $\beta+$ IVS-I-110, $\beta 0$ IVS-I-1, $\beta+$ IVS-I-6. ${ }^{2}$

A apresentação genética será o principal determinante do grau de intensidade da doença, que varia da forma minor (a mais branda, heterozigota) à major (a mais severa, homozigota), existindo também a forma intermediária. ${ }^{3}$

O paciente talassêmico do tipo beta desenvolve anemia por produzir com deficiência essa parcela globínica e promover o acúmulo e precipitação das cadeias alfa nos eritroblastos e consequente apoptose. ${ }^{3}$ A incidência mundial estimada de indivíduos beta-talassêmicos sintomáticos é de 1 em 100.000, somando-se todos os tipos da doença. ${ }^{4}$

A beta-talassemia major (homozigótica) é uma condição rara que ocasiona grave anemia pela ausência de síntese da cadeia beta da hemoglobina. ${ }^{5}$ É datado de 1925 o primeiro registro e descrição clínico-hematológica de beta-talassemia major, feito pelos pediatras Thomas Cooley e Pearl Lee. Nessa investigação foi atribuído um possível caráter genético de povos do Mediterrâneo à doença, já que os pacientes estudados possuíam ascendência grega e italiana. ${ }^{6}$ Por esses motivos, a talassemia major ficou conhecida como anemia de Cooley ou do Mediterrâneo e em 1940 o nome passou a ser talassemia major ('thalassa', que significa mar e 'haima', doença do sangue).

A dispersão dos genes responsabilizados pelas anemias hereditárias no Brasil ocorreu através do processo de colonização e consequente formação étnica de nossa população. Segundo dados do Ministério da Saúde, entre 2013 e 2015, foram registrados 593 casos de talassemia acompanhados em centros de hemoterapia e assistência hematológica. $\mathrm{O}$ percentual de pessoas com as formas graves (beta major e intermediária) distribuídas nas regiões brasileiras foi: Sudeste (60\%), Nordeste (17\%), Sul (10,4\%), Centro-Oeste (7,3\%) e Norte $(5,3 \%) .{ }^{6}$ Devido à escassez de trabalhos publicados sobre fatores prognósticos, tratamento tardio e índices de casos sem diagnóstico fechado, não se pode inferir sobre a subnotificação em casos de beta-talassemia no estado do Ceará.

Além dos achados clínicos sugestivos, a suspeição da talassemia beta major se inicia através do exame de hemograma, onde se revela uma anemia microcítica e hipocrômica. O esfregaço característico da forma major apresenta anisocitose, poiquilocitose e numerosos eritrócitos diminutos.

A eletroforese qualitativa e quantitativa de hemoglobina é um importante exame de triagem nesses casos. A técnica em $\mathrm{pH}$ alcalino permite a separação das hemoglobinas $\mathrm{HbA}, \mathrm{HbF}$ e $\mathrm{HbA} 2$ das $\mathrm{HbC}$ e $\mathrm{HbS}$.

O tratamento dos pacientes que apresentam beta-talassemia major é baseado em esquema transfusional seriado e aporte medicamentoso para quelação de ferro excedente. ${ }^{3,7,8}$ Quando inadequadamente tratados ou não tratados podem apresentar deformidades ósseas, alteração importante no crescimento, insuficiência cardíaca, cirrose hepática e falências endócrinas. ${ }^{5,9-11}$

\section{METODOLOGIA}

Realizou-se estudo descritivo do tipo relato de caso de uma paciente acompanhada no Hospital Escola Santa Casa de Misericórdia de Sobral, Ceará e ambulatório de Hematologia - HEMOCE. O projeto foi aprovado pelo Comitê de Ética e Pesquisa da Universidade Estadual Vale do Acaraú (UEVA).

\section{RELATO DE CASO}

Paciente com 6 anos e 10 meses de idade, sexo feminino, natural e procedente do município de Granja - CE, residente no distrito de Timonha. Nascida a termo, parto vaginal domiciliar, peso de $3080 \mathrm{~g}$ e sem dados confiáveis para comprimento. Pais primos em segundo grau.

Deu entrada na UTI pediátrica da Santa Casa de Misericórdia de Sobral, apresentando rebaixamento do nível de consciência (escala de coma de Glasgow = 9/15), intensa palidez cutâneo-mucosa e hipotensão. O hemograma evidenciava hemoglobina de $1,9 \mathrm{~g} / \mathrm{dL}$.

História familiar marcada por anemia, diagnosticada, porém subtratada, nos pais, tios e primos paternos e maternos.

Apresentava história de três internamentos anteriores por quadro semelhante, nos quais apresentava também tosse, dor abdominal, cefaleia e anasarca.

No primeiro internamento, aos 4 anos e 11 meses, foram aventadas como principais hipóteses diagnósticas: leishmaniose visceral (descartada por critérios clínicos e laboratoriais) e anemia hemolítica autoimune, subsidiada por teste de Coombs direto positivo. A paciente foi encaminhada ao ambulatório de hematologia pediátrica por melhora clínica após corticoterapia e hemotransfusão, porém, não deu seguimento ao tratamento e investigação diagnóstica por limitações financeiras da família para translado para Sobral e dificuldades de acesso à medicação em localidade de residência. Após dois anos, voltou a ser admitida por duas 
vezes em serviços de emergência por quadro de vômitos, dor abdominal, cefaleia intensa e febre.

Ao exame físico admissional, apresentava-se hipocorada $(4+/ 4+)$, ictérica $(2+/ 4+)$, taquipnéica (frequência respiratória $=40 \mathrm{irpm}$ ), taquicárdica (frequência cardíaca $=110$ bpm) massa corporal $=18 \mathrm{Kg}$ (entre o percentil 3 e 15), estatura $=105 \mathrm{~cm}(<$ percentil 3); Índice de Massa Corpórea = 16,3 (percentil entre 50 e 85). Macrocefalia (perímetro cefálico de $58 \mathrm{~cm}$ ), ponte nasal achatada (Figura 1A) e hipertrofia gengival (Figura 1B). Ausculta pulmonar: murmúrio vesicular universal sem ruídos adventícios; ausculta cardíaca: ritmo cardíaco regular, em dois tempos, bulhas hiperfonéticas com sopros holossistólicos audíveis em todos os focos grau III-IV/VI; abdome globoso, ruídos hidroaéreos presentes, hepatoesplenomegalia (fígado palpável $5 \mathrm{~cm}$ abaixo do rebordo costal direito e baço palpável $9 \mathrm{~cm}$ abaixo do rebordo costal esquerdo) (Figura 1C).

Os exames laboratoriais realizados durante a investigação, evidenciaram níveis de hemoglobina menores que $7 \mathrm{~g} / \mathrm{dL}$ com discretas elevações após esquemas transfusionais; morfologia eritrocitária demonstrando anisocitose, microcitose, policromasia, hipocromia, poiquilocitose e reticulocitose (Tabela 1). Realizado estudo de ferritina sérica e eletroforese de hemoglobina por HPLC (High Performance Liquid Chromatography) da criança e dos pais (Tabelas 2, 3 e 4).

Realizada ecografia abdominal que confirmou a hepatoesplenomegalia sem evidências de litíase biliar. Ecocardiografia evidenciou insuficiência mitral e tricúspide de graus discretos. A tomografia de crânio demonstrou hipertrofia da calota craniana $(3,5 \mathrm{~cm})$ prioritariamente decorrente de hiperplasia osteocondensante da medula (displasia óssea).

Após alta hospitalar, a paciente seguiu em acompanhamento ambulatorial periódico e multiprofissional (com endocrinologista, geneticista, odontólogo e psicólogo). Encontra-se clinicamente estável, em regime de hipertransfusão a cada duas semanas e tratamento quelante de ferro com deferasirox. Aguarda estudo genotípico e pesquisa de mutações no gene da globina $\beta$.

Figura 1. A: hipertrofia gengival e ponte nasal achatada; B: hipertrofia gengival; C: hepatoesplenomegalia.

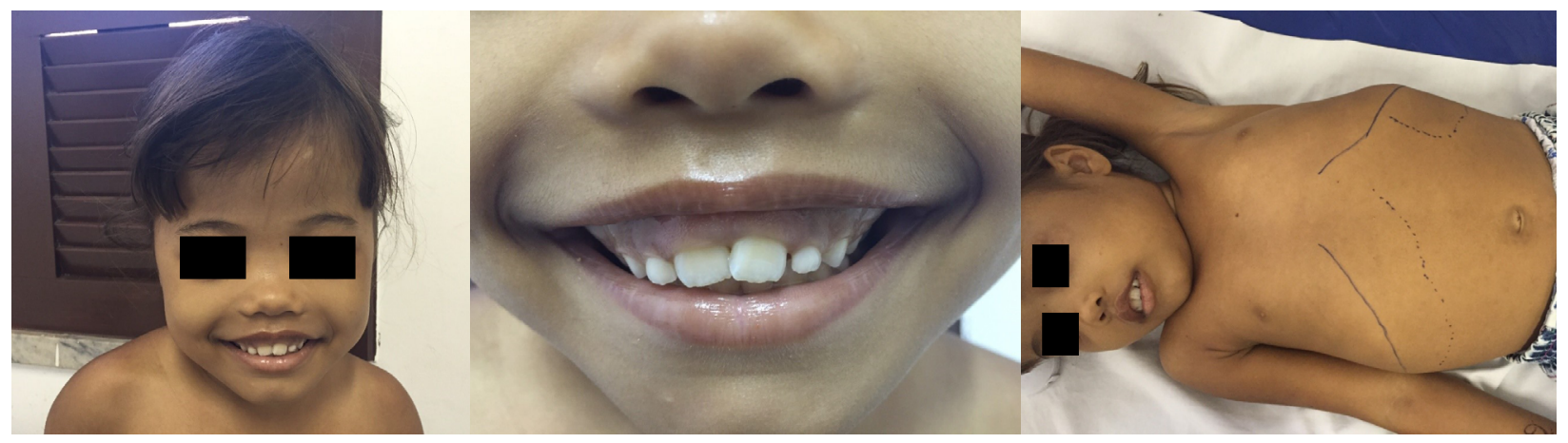

Tabela 1. Perfis hematimétricos conforme as internações.

\begin{tabular}{llllllll}
\hline Internação & Hemácias (10 $/ \mathbf{U I})$ & $\mathbf{H b}(\mathbf{g} / \mathbf{d L})$ & Ht (\%) & VCM (fl) & HCM (pg) & CHCM (g/dL) & RDW (\%) \\
\hline Primeira & 2,2 & 5,6 & 18,6 & 85 & 25,4 & 29,9 & 13,8 \\
Segunda & 3,3 & 8,8 & 26,3 & 80,6 & 33,3 & 26,8 & 20,2 \\
Terceira & 0,9 & 1,9 & 6,5 & 75 & 22,2 & 29,6 & 28,9 \\
\hline
\end{tabular}

Hb: dosagem da hemoglobina; Ht: hematócrito; VCM: volume corpuscular médio; HCM: hemoglobina corpuscular média; CHCM: concentração da hemoglobina corpuscular média; RDW: red cell distribution width.

Tabela 2. Estudo de eletroforese de hemoglobina (por HPLC) da criança, do pai e da mãe.

\begin{tabular}{ccccc}
\hline Paciente & A (\%) & A2 $(\mathbf{\%})$ & F (\%) & Diagnóstico \\
\hline Criança & 43,6 & 0 & 54 & Talassemia major \\
Mãe & 93,3 & 6 & 0,7 & Talassemia minor \\
Pai & 94,6 & 5,4 & 0 & Talassemia minor \\
\hline
\end{tabular}


Tabela 3. Perfis hematimétricos da criança, da mãe e do pai.

\begin{tabular}{llllllll}
\hline Paciente & Hemácias $\mathbf{( 1 0} / \mathbf{U I})$ & Hb (g/dL) & Ht (\%) & VCM (fl) & HCM (pg) & CHCM (g/dL) & RDW (\%) \\
\hline Criança & 0,9 & 1,9 & 6,5 & 75 & 22,2 & 29,6 & 28,9 \\
Mãe & 5,4 & 9,7 & 30,9 & 57 & 18 & 31,5 & 16,5 \\
Pai & 7,2 & 13,3 & 47 & 65 & 18,4 & 28,3 & 15,9 \\
\hline
\end{tabular}

Hb: dosagem da hemoglobina; Ht: hematócrito; VCM: volume corpuscular médio; HCM: hemoglobina corpuscular média; CHCM: concentração da hemoglobina corpuscular média; RDW: red cell distribution width.

Tabela 4. Estudo do Ferro e ferritina séricos e reticulócitos do paciente.

\begin{tabular}{lll}
\hline & Paciente & Valores de referência \\
\hline Ferro sérico $(\mu \mathrm{g} / \mathrm{dl})$ & 208,8 & $35-150$ \\
Ferritina $(\mathrm{ng} / \mathrm{ml})$ & 2272,4 & $0-291$ \\
Reticulocitos $(\%)$ & 4,3 & $0,5-1,5$ \\
\hline
\end{tabular}

\section{DISCUSSÃO}

Em nosso contexto médico-social, onde ainda há grande prevalência da anemia carencial, a detecção de hemoglobinopatias e inclusão das mesmas na investigação clínica é importante. Quando se trata do padrão microcítico e hipocrômico, incluímos as talassemias no conjunto de possibilidades diagnósticas, já que aproximadamente 2,7 milhões de brasileiros apresentam a forma minor. ${ }^{12}$

A presença portuguesa no território cearense é vista como fator importante para o surgimento de casos de talassemia. Rocha et $a l^{2}$ conduziu estudo para traçar o perfil das mutações encontradas em pacientes beta-talassêmicos da cidade de Fortaleza - Ceará, encontrando em $35,7 \%$ do grupo o tipo IVS-I-6, relevante em população da região central de Portugal.

Para se realizar o diagnóstico de beta talassemia major são realizados exames laboratoriais de triagem e de confirmação, em complementação com os achados clínicos do paciente. ${ }^{13}$

Dentre os exames de triagem estão: hemograma, análise morfológica de hemácias, estudo do ferro e ferritina séricos, eletroforese de hemoglobina em $\mathrm{pH}$ alcalino e resistência osmótica em solução de cloreto de sódio a $0,36 \%$. Para confirmação, temos: eletroforese em pH ácido, isoeletroforese, cromatografia líquida de alta pressão (HPLC) e estudo molecular de DNA. ${ }^{13}$

Os achados laboratoriais que sugerem hemólise extravascular, como hiperbilirrubinemia indireta, elevação do LDH (lactato desidrogenase) e redução da haptoglobina estão presentes. A análise morfológica das hemácias do sangue periférico evidencia: anisopoiquilocitose, presença de leptócitos e eritroblastose.

A hemoglobina total, em geral, varia entre 3 e $7 \mathrm{~g} / \mathrm{dL}$. O padrão de hemoglobinas é variável, sendo comum o aumento de $\mathrm{Hb} F(60-90 \%)$ e $\mathrm{Hb}$ A2 dentro da normalidade ou elevada. A presença de $\mathrm{Hb} \mathrm{A}$ é relacionada à produção parcial das cadeias beta.

O diagnóstico tardio pode trazer múltiplas complicações. No caso citado, a paciente apresentava alterações pônderoestaturais, deformidades ósseas, hepatoesplenomegalia, insuficiência cardíaca e outros comprometimentos clínicos decorrentes do grau severo de anemia.

São apontados como fatores limitantes para a adesão ao tratamento, o desconhecimento por parte dos familiares de informações básicas sobre a doença e a carência na investigação genética dos pais. ${ }^{14}$ Portanto, evidenciamos a importância do diagnóstico precoce, visando a minimização da morbimortalidade dos pacientes que apresentem a forma grave de talassemia beta, sendo fundamental a participação das famílias para o seguimento clínico e para o devido aconselhamento genético.

\section{CONSIDERAÇÕES FINAIS}

A inclusão das hemoglobinopatias no Programa Nacional de Triagem Neonatal, através da portaria 822/01 do Ministério da Saúde representou uma grande aquisição à saúde do país..$^{15}$ Porém, mesmo com a sua implementação, ainda percebemos lacunas na abrangência de populações provenientes de localidades distantes de grandes centros, assim como limitações no aporte para tratamento de pessoas talassêmicas e para aconselhamento genético.

Nota-se também pequeno número de estudos nacionais que tratem das influências socioculturais que limitam a possibilidade de diagnóstico e a adesão ao tratamento dos pacientes talassêmicos.

Vale destacar, por fim, que para o seguimento clínico destes pacientes é necessário acompanhamento multiprofissional e suporte social para evitar evasões e consequentes agravamentos. 


\section{REFERÊNCIAS}

1. Higgs DR, Engel JD, Stamatoyannopoulos G. Thalassaemia. Lancet. 2012;379(9813):373-83.

2. Rocha LB, Martins MF, Gonçalves RP. Distribuição das mutações da $\beta$-talassemia em Fortaleza, Ceará. J Bras Patol Med Lab. 2010;46(6):437-41.

3. Galanello R, Origa R. Beta-thalassemia. Orphanet J Rare Dis. 2010;21;5:11.

4. Sankaran VG, Weiss MJ. Anemia: progress in molecular mechanisms and therapies. Nat Med. 2015;21(3):221-30.

5. Trigo LA, Surita FG, Parpinelli MA, Pereira BG, Fertrin KY, Costa ML. Talassemia beta maior e gestação na adolescência: relato de dois casos. Rev Bras Ginecol Obstet. 2015;37(6):291-6.

6. Brasil. Ministério da Saúde. Secretaria de Atenção à Saúde. Departamento de Atenção Especializada e Temática. Orientações para diagnóstico e tratamento das Talassemias Beta. Brasília: Ministério da Saúde; 2016.

7. Cooley TB, Lee PA. A series of cases of splenomegaly in children with anemia and peculiar bone changes. Transactions of the American Pediatric Society. 1925;37:29-30.

8. Marsella M, Borgna-Pignatti C. Transfusional iron overload and iron chelation therapy in thalassemia major and sickle cell disease. Hematol Oncol Clin North Am. 2014;28(4):703-27.
9. Toumba M, Sergis A, Kanaris C, Skordis N. Endocrine complications in patients with Thalassaemia Major. Pediatr Endocrinol Rev. 2007;5(2):642-8.

10. Borgna-Pignatti C, Gamberini MR. Complications of thalassemia major and their treatment. Expert Rev Hematol. 2011; 4(3):353-66.

11. Rachmilewitz EA, Giardina PJ. How I treat thalassemia. Blood. 2011;118(13):3479-88.

12. Reis PR, Penna KG, Araújo LM, Mesquita MM, Castro FS, Balestra FA. Prevalência de hemoglobinopatias e talassemias em crianças de 6 meses a 7 anos de idade no laboratório escola do departamento de biomedicina (CBB)-UCG. Rev Bras Anal Clin. 2005;37(1):3-5.

13. Vargas SP, Yamagushi UM. Diagnóstico laboratorial para talassemias. Revista Saúde e Pesquisa. 2008;1(1):85-8.

14. Melo-Reis PR, Araújo LM, Dias-Penna KG, Mesquita MM, Castro FS, Costa SH. A importância do diagnóstico precoce na prevenção das anemias hereditárias Rev Bras Hematol Hemoter. 2006;28(2):149-52.

15. Ramalho AS, Magna LA, Paiva-E-Silva RB. A Portaria $n^{\circ} 822 / 01$ do Ministério da Saúde e as peculiaridades das hemoglobinopatias em saúde pública no Brasil. Cad Saude Publica. 2003;19(4):1195-9.

\section{Como citar:}

Freire IA, Deolindo AM, Sanches MS, Arcanjo FP. $\beta$-Talassemia major: um relato de caso. Rev Med UFC. 2019 abr-jun;59(2):66-70. 